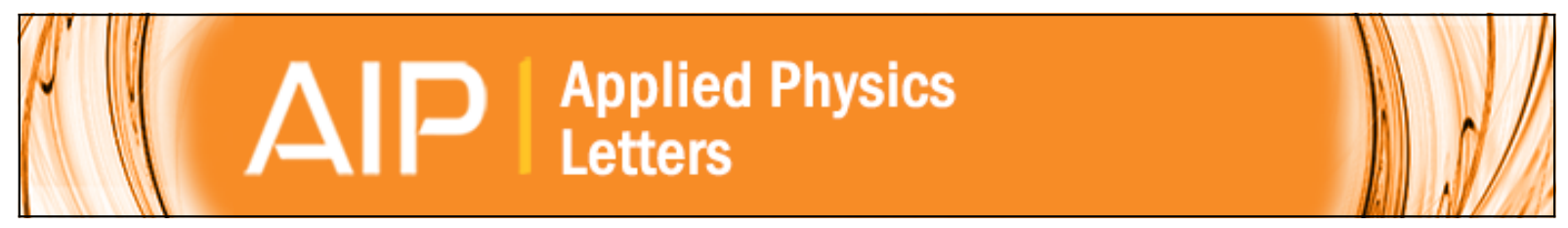

\title{
Fabrication of $\mathbf{n}$-type carbon nanotube field-effect transistors by Al doping
}

Hwangyou Oh, Ju-Jin Kim, Woon Song, Sunkyung Moon, Nam Kim, Jinhee Kim, and Noejung Park

Citation: Applied Physics Letters 88, 103503 (2006); doi: 10.1063/1.2183818

View online: http://dx.doi.org/10.1063/1.2183818

View Table of Contents: http://scitation.aip.org/content/aip/journal/apl/88/10?ver=pdfcov

Published by the AIP Publishing

\section{Articles you may be interested in}

Comparison of sensitivities of carbon nanotube field-effect transistor biosensors with and without top metal gate J. Appl. Phys. 104, 104304 (2008); 10.1063/1.3020688

One-step grown suspended $\mathrm{n}$-type semiconducting single wall carbon nanotube field effect transistors with carbon nanotube electrodes

Appl. Phys. Lett. 92, 043110 (2008); 10.1063/1.2838736

Poly( 3,3 -didodecylquarterthiophene) field effect transistors with single-walled carbon nanotube based source and drain electrodes

Appl. Phys. Lett. 91, 223512 (2007); 10.1063/1.2806234

Time-related conversion of the carbon nanotube field effect transistor

Appl. Phys. Lett. 89, 233507 (2006); 10.1063/1.2402218

Mechanism of $\mathrm{N} \mathrm{O} 2$ detection in carbon nanotube field effect transistor chemical sensors

Appl. Phys. Lett. 88, 123112 (2006); 10.1063/1.2187510

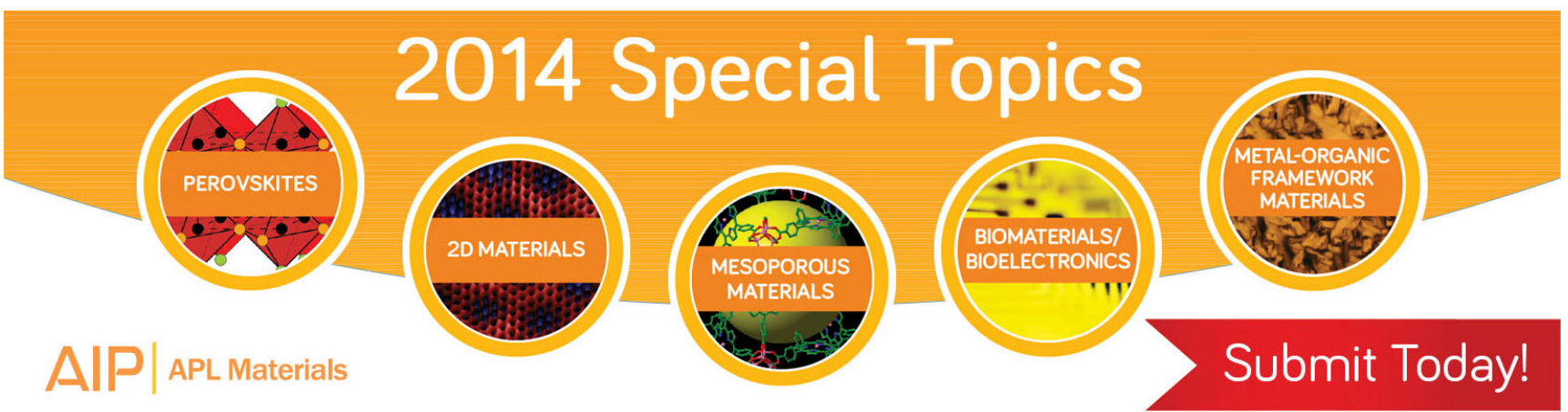




\title{
Fabrication of $n$-type carbon nanotube field-effect transistors by Al doping
}

\author{
Hwangyou Oh and Ju-Jin Kim \\ Department of Physics, Chonbuk National University, Jeonju 561-756, Korea \\ Woon Song, Sunkyung Moon, Nam Kim, and Jinhee Kim ${ }^{\text {a) }}$ \\ Electronic Devices Group, Korea Research Institute of Standards and Science, Daejeon 305-600, Korea \\ Noejung Park \\ Department of Applied Physics, Dankook University, Seoul 140-714, Korea
}

(Received 11 August 2005; accepted 1 February 2006; published online 8 March 2006)

\begin{abstract}
We report the effect of an $\mathrm{Al}$ layer, covering the central part of the nanotube channel, on the electrical transport properties of carbon nanotube field-effect transistors (CNFETs). The CNFETs, consisting of single-walled carbon nanotube or double-walled carbon nanotube between two Pd electrodes on top of $\mathrm{SiO}_{2}$ layer, which showed $p$-type or ambipolar transport behaviors, exhibit clear $n$-type characteristics after the $\mathrm{Al}$ deposition. We ascribe such conversions into $n$-type behaviors to the electron doping in the Al-covered nanotube region, which results in the bending of the nanotube bands nearby the edges of the $\mathrm{Al}$ layer. This technique, $\mathrm{Al}$ deposition under a high vacuum, may give rise to a practical fabrication method for the $n$-type CNFET, which may enable us to develop complementary logic nanotube electronic devices. (C) 2006 American Institute of Physics.
\end{abstract}

[DOI: $10.1063 / 1.2183818]$

A carbon nanotube $(\mathrm{CNT})$, a graphitic tube with nanometer-scale diameter, ${ }^{1}$ has strong mechanical stiffness ${ }^{2}$ and excellent electrical transport properties. ${ }^{3-5}$ Such advantageous material properties may lead to many potential applications; nanotube electron emitter for flat-panel displays with extremely low-power consumptions, ${ }^{6}$ highly sensitive chemical sensors, ${ }^{7}$ and nanoscale electronics devices, such as memory and logic circuits. ${ }^{8-10}$ These nanoelectronics may give rise to future breakthroughs in the electronics industry when the Si-based semiconductor technology reaches its scaling limit.

Since the first CNT field-effect transistor (CNFET) (Refs. 11 and 12) was developed, quite a few studies have been devoted to understanding and controlling the conduction type of the CNFET. It has been known that most of the CNFETs are likely to show $p$-type characteristics, which is either due to the hole doping by the environmental oxygen or a lower Schottky barrier for hole current at the metal-CNT contact. In order to achieve operations similar to complementary metal-oxide-semiconductor devices, well-defined $n$-type transistors as well as $p$-type ones are needed. A few successes in the fabrication of the $n$-type CNFET have utilized potassium doping in the CNT channel ${ }^{13-16}$ or a calcium contact as an electrode with a low work function. ${ }^{17}$ However, these alkali elements are chemically very active, and usually very hard to control. Thus more practical and reliable method to fabricate $n$-type CNFET is required.

In this work, we show that an $\mathrm{Al}$ layer on top of a CNT, deposited by sputtering under a high vacuum, gives rise to $n$-type transport properties in the CNFET. We first fabricated a CNFET with an individual single-walled carbon nanotube (SWNT) or double-walled carbon nanotube (DWNT) between Pd electrodes. Before $\mathrm{Al}$ deposition, the devices show $p$-type or ambipolar behaviors, which may be due to the Fermi-level alignment at the contacts for SWNT or the small

\footnotetext{
a) Author to whom correspondence should be addressed; electronic mail:
} jinhee@kriss.re.kr band gap for DWNT. However, once the Al layer was deposited on the CNT, the device became a clear $n$-type CNFET. We describe these changes in the transport behaviors with the band bending, caused by the electron doping in the CNT region under the $\mathrm{Al}$ layer.

For our experiment, high-purity DWNTs and SWNTs were synthesized by a hydrogen arc discharge method. ${ }^{18}$ Atomic force microscope (AFM) study indicated that the diameters of DWNTs were in the range of $2-4 \mathrm{~nm}$ and those of SWNTs were 1-2 nm. A droplet of dichloroethane containing CNTs was spun over a Si substrate with a $300 \mathrm{~nm}$ thick thermally grown $\mathrm{SiO}_{2}$ layer. Individual CNTs were located by AFM and conventional electron-beam lithography was used to generate electrical lead patterns onto a selected CNT. To form source and drain electrodes, $30 \mathrm{~nm}$ of Pd was deposited by magnetron sputtering at a base pressure of $10^{-8}$ Torr, followed by a standard lift-off process. Then, the electrical transport properties were measured at room temperature in the vacuum condition. A back gate was used for gating. After confirming the FET behavior of the device, we have generated the second pattern for $\mathrm{Al}$ deposition in the middle of the CNT. A $30 \mathrm{~nm}$ thick Al film was deposited by the same method as Pd. Then, the electrical transport properties of the device, between two Pd electrodes, were measured at room temperature in the vacuum condition.

The schematic device structure is shown in Fig. 1, where $L$ is the separation between the source and drain electrodes

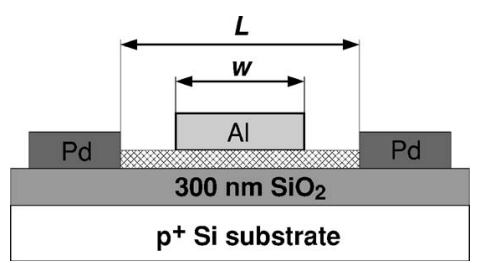

FIG. 1. Schematic device structure. $L$ is the distance between the Pd electrodes and $w$ is the width of the Al layer. Thickness of Pd and Al film is about $30 \mathrm{~nm}$. 

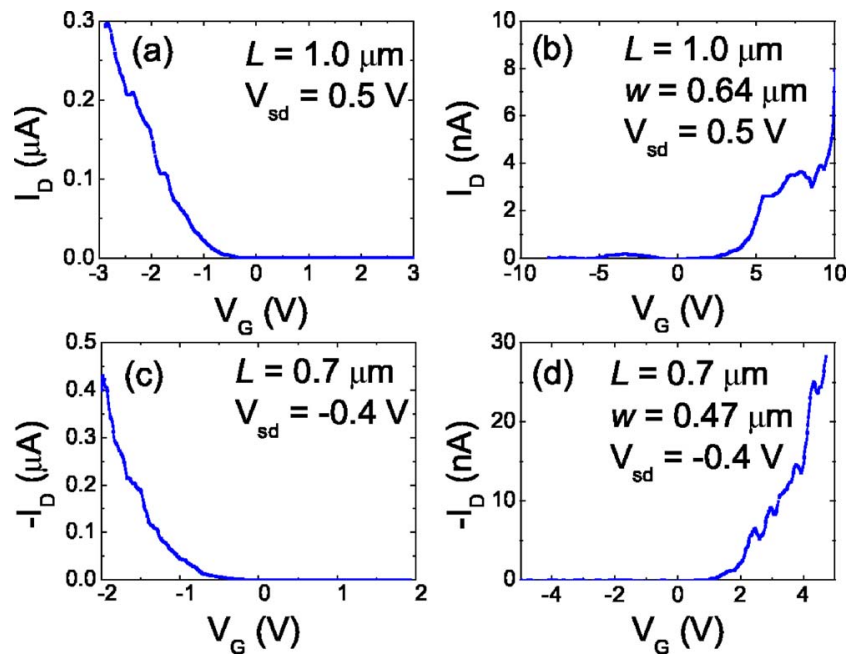

FIG. 2. The gate modulation characteristics for sample S1 (a) before and (b) after the Al layer deposition. The same characteristics for sample S2 (c) before and (d) after the Al layer deposition. Through the AFM measurement, diameters of the nanotubes are determined to be about 1.2 and $1.5 \mathrm{~nm}$ for sample S1 and S2, respectively.

and $w$ is the width of the $\mathrm{Al}$ layer. We have found that a high-energy deposition, such as sputtering, at a high vacuum $\left(10^{-8}\right.$ Torr $)$ is required to achieve $n$-type transport behaviors. For a comparison, we have also performed the same experiment with the Al layer deposited by an electron-beam deposition method at the base pressure of $10^{-6}$ Torr. We have found that such a method did not lead to any noticeable change of the field effect transistor (FET) behavior; original $p$-type CNFET exhibited $p$-type behavior even after the deposition of Al layer. Below, we report the three samples which exhibit $p$ - to $n$-type conversion. For samples S1, $L$ $=1.0 \mu \mathrm{m}$ and $w=0.64 \mu \mathrm{m}$, for sample $\mathrm{S} 2, L=0.70 \mu \mathrm{m}$ and $w=0.47 \mu \mathrm{m}$, and for sample S3, $L=5.3 \mu \mathrm{m}$ and $w$ $=1.3 \mu \mathrm{m}$.

Figure 2(a) shows the gate modulation of sample S1 without Al deposition. An apparent $p$-type FET behavior is observed. The conductance at the bias voltage of $V_{s d}$ $=0.5 \mathrm{~V}$ is about $150 \mathrm{nA} / \mathrm{V}$. After the deposition of the Al film in the middle of CNT, the transfer characteristics change into a $n$-type FET, as shown in Fig. 2(b). The conductance at the bias voltage of $V_{s d}=0.5 \mathrm{~V}$ is about $1 \mathrm{nA} / \mathrm{V}$, which means a two orders of magnitude decrease in the conductance by the Al deposition. Such a conductance decrease after the $\mathrm{Al}$ deposition was commonly observed in other samples of ours as well as in other's work. ${ }^{19}$ Similar behavior was also observed for the sample S2. Figures 2(c) and 2(d) show the gate modulations of sample S2. For sample $\mathrm{S} 2$, the conductance decreases by about one order of magnitude after the $\mathrm{Al}$ deposition.

Sample S3 exhibited ambipolar behavior before the Al deposition as shown in Fig. 3(a). Such ambipolar behavior is commonly observed in a DWNT or a large-diameter SWNT (Refs. 20 and 21) and is attributed to the small band gap of the large-diameter CNT. Through the AFM measurement, we have found that the diameter of the CNT in sample S3 was about $3.0 \mathrm{~nm}$, larger than the diameters $(\sim 1.5 \mathrm{~nm})$ of the CNTs in samples S1 and S2. Like the other two samples, sample S3 also exhibits a transition to a $n$-type CNFET after the $\mathrm{Al}$ deposition. Figure 3 shows the gate modulations of the sample S3. Before the Al deposition [Fig. 3(a)], this device
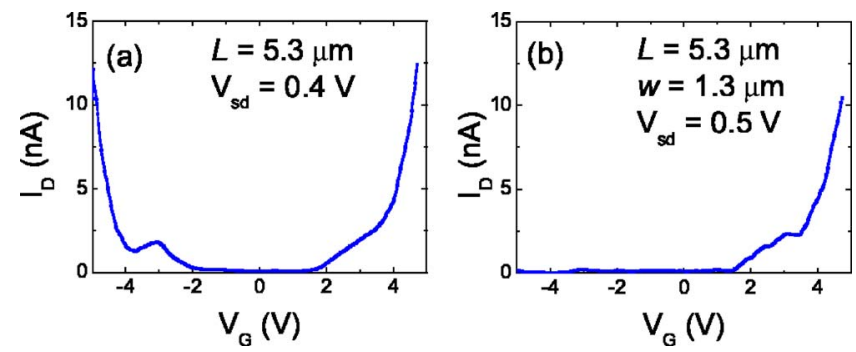

FIG. 3. The gate modulation characteristics for sample S3 (a) before and (b) after Al layer deposition. The diameter of the nanotube is about $3.0 \mathrm{~nm}$.

showed an ambipolar behavior. After the Al film deposition, the $p$-channel current was suppressed considerably, while the $n$-channel current was negligibly affected, as shown in Fig. 3(b).

As we mentioned before, when the Al layer was deposited with the electron-beam deposition method at a relatively low $\left(\sim 10^{-6}\right.$ Torr $)$ base pressure, no $p$ - to $n$-type transition was observed. The absence of the type conversion can be explained by the oxidation of the Al layer during or after the film deposition. Recent theoretical study shows that the work function of the oxidized $\mathrm{Al}$ surface is close to that of $\mathrm{Au}$ and, therefore, the oxidized $\mathrm{Al}$ particles are likely to induce hole doping, rather than the electron doping, in the CNT. ${ }^{22}$ This means that the Al particle, once oxidized, may lead to $p$-type behavior in a CNFET, thus no type conversion would occur. Because we could not observe type conversion in CNFETs, where the $\mathrm{Al}$ layer was deposited by electron-beam evaporation, we suspect that Al layers deposited by electron-beam evaporation oxidize more than those deposited by sputtering. In the electron-beam evaporation process, $\mathrm{Al}$ particles can be substantially oxidized during evaporation under a low vacuum condition $\left(10^{-6}\right.$ Torr $)$ and low deposition rate $(\sim 1 \mathrm{~nm} / \mathrm{s})$. Moreover, oxidation of Al layer may occur even after the evaporation process because non-negligible spacing could be formed between $\mathrm{CNT}$ and Al film, which may result in the oxidation of the Al layer after deposition. Therefore, we suggest that high-energy evaporation, such as sputtering, under a high vacuum would be crucial to growing an Al layer without oxidation, and in turn to achieve $n$-type CNFETs.

We now suggest a theoretical explanation on the type conversion of CNFET with Al deposition. It is well known that the $p$-type operations of CNFET (samples S1 and S2) are largely attributed to the large work function of the $\mathrm{Pd}$ electrodes. When the electrodes are large work-function metals, such as Pd or Au, the Fermi level of the electrodes is located close to the valence-band edge of the CNT, as shown in the band diagram of Fig. 4(a), resulting in a $p$-type CNFET. $^{22}$ However, the ambipolar behavior of sample S3 [Fig. 3(a)] is likely due to the smaller band gap of the large diameter CNT. The channel length of sample S3 is much longer than those of samples S1 and S2. With this longer

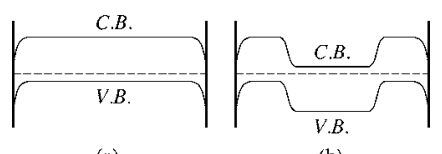

(a)

(b)

FIG. 4. The band diagrams along the axis of the nanotube (a) before and (b) after the Al layer deposition. C.B. and V.B. represent the conduction band and the valence band, respectively. The horizontal dashed line represents the Fermi level. 
channel length, the CNT is more likely to be in a diffusive conduction regime ${ }^{5}$ and the CNT channel may contribute more to the resistance than the metal-CNT contacts. ${ }^{23}$

The $n$-type behaviors, as shown in Figs. 2(b), 2(d), and $3(\mathrm{~b})$, could be ascribed to the electron doping by the deposited $\mathrm{Al}$ layers. It has been shown that electronic charge transfers from Al layer to the CNT when unoxidized Al particles are in the vicinity of the $\mathrm{CNT}^{22}$ The overall band diagram after the $\mathrm{Al}$ deposition is described in Fig. 4(b). We note that the Fermi level is aligned at the valence-band edge near the $\mathrm{Pd}$ electrode, while it is at the conduction-band edge under the deposited Al layers. Thus, there must be a sharp band bending near the edges of the $\mathrm{Al}$ layer. For electrons to flow through the CNT, they need to be thermally excited or to experience interband tunneling near the edges of the $\mathrm{Al}$ covered region. If the deposited $\mathrm{Al}$ particles did not contaminate the metal-CNT contact, the Schottky barriers described in Figs. 4(a) and 4(b) would not change upon the Al deposition. Thus, the decreased current with deposited Al layer, as shown in Figs. 2(b) and 2(d), should be ascribed to the additional resistance near the edges of the $\mathrm{Al}$ layers.

In summary, we fabricated CNFETs with an individual CNT between two Pd electrodes and measured their transport properties. While most devices showed $p$-type transport behaviors, some large-diameter CNFETs showed ambipolar behaviors. After covering the CNT channel region with the $30 \mathrm{~nm}$ thick Al layer deposited by sputtering at a high vacuum, we achieved $n$-type transport behaviors in the aforementioned CNFETs. We explained such conversion from $p$-type to $n$-type behavior in terms of the bending of the conduction and valence bands, which originates from the electron doping in the $\mathrm{CNT}$ region under the $\mathrm{Al}$ layer. We suggest that the deposition of $\mathrm{Al}$ layers without oxidation would be a key technique to achieve $n$-type CNFETs, and could pave the way to a more practical fabrication method for $n$-type CNFETs.

This work was supported by the Center for Nanotubes and Nanostructured Composites and Ministry of Science and Technology of Korea. One of the authors (J.J.K.) acknowledges the support by Tera-level Nanodevice project. The au- thors are also grateful to C. Lee for providing high-purity DWNTs.

${ }^{1}$ S. Iijima, Nature (London) 354, 56 (1991).

${ }^{2}$ J.-P. Salvetat, G. Andrew, D. Briggs, J.-M. Bonard, R. R. Bacsa, A. J. Kulik, T. Stöckli, N. A. Burnham, and L. Forró, Phys. Rev. Lett. 82, 944 (1999).

${ }^{3}$ S. Frank, P. Poncharal, Z. L. Wang, and W. A. De Heer, Science 280, 1744 (1998).

${ }^{4}$ C. T. White and T. N. Todorov, Nature (London) 393, 240 (1998).

${ }^{5}$ A. Javey, J. Guo, Q. Wang, M. Lundstrom, and H. Dai, Nature (London) 424, 654 (2003).

${ }^{6}$ J. E. Jung, J. H. Choi, Y. J. Park, H. W. Lee, Y. W. Jin, D. S. Chung, S. H. Park, J. E. Jang, S. Y. Hwang, T. Y. Ko, Y. S. Choi, S. H. Cho, C. G. Lee, J. H. You, N. S. Lee, J. B. Yoo, and J. M. Kim, J. Vac. Sci. Technol. B 21, 375 (2003).

${ }^{7}$ J. Kong, N. R. Franklin, C. Zhou, M. G. Chapline, S. Peng, K. Cho, and H. Dai, Science 287, 622 (2000).

${ }^{8}$ T. Rueckes, K. Kim, E. Joselevich, G. Y. Tseng, C.-L. Cheung, and C. M. Lieber, Science 289, 94 (2000).

${ }^{9}$ A. Bachtold, P. Hadley, T. Nakanishi, and C. Dekker, Science 294, 1317 (2001).

${ }^{10}$ V. Derycke, R. Martel, J. Appenzeller, and P. Avouris, Nano Lett. 1, 453 (2001).

${ }^{11}$ S. J. Tans, A. R. M. Verschueren, and C. Dekker, Nature (London) 393, 49 (1998).

${ }^{12}$ R. Martel, T. Schmidt, H. R. Shea, T. Hertel, and P. Avouris, Appl. Phys. Lett. 73, 2447 (1998)

${ }^{13}$ M. Bockrath, J. Hone, A. Zettl, and P. L. McEuen, Phys. Rev. B 61, R10606 (2000).

${ }^{14}$ V. Derycke, R. Martel, J. Appenzeller, and P. Avouris, Appl. Phys. Lett. 80, 2773 (2002)

${ }^{15}$ J. Kong, J. Cao, H. Dai, and E. Anderson, Appl. Phys. Lett. 80, 73 (2002).

${ }^{16}$ A. Javey, R. Tu, D. B. Farmer, J. Guo, R. G. Gordon, and H. Dai, Nano Lett. 5, 345 (2005).

${ }^{17}$ Y. Nosho, Y. Ohno, S. Kishimoto, and T. Mizutani, Appl. Phys. Lett. 86, 073105 (2005)

${ }^{18}$ S. C. Lyu, B. C. Liu, S. H. Lee, C. Y. Park, H. K. Kang, C.-W. Yang, and C. J. Lee, J. Phys. Chem. B 108, 2192 (2004).

${ }^{19}$ B.-K. Kim, N. Park, P. S. Na, H.-M. So, J.-J. Kim, H. Kim, K.-J. Kong, H. Chang, B.-H. Ryu, Y. Choi, and J. Lee, Nanotechnology (to be published).

${ }^{20}$ T. Shimada, T. Sugai, Y. Ohno, S. Kishimoto, T. Mizutani, H. Yoshida, T. Okazaki, and H. Shinohara, Appl. Phys. Lett. 84, 2412 (2004).

${ }^{21}$ A. Javey, M. Shim, and H. Dai, Appl. Phys. Lett. 80, 1064 (2002).

${ }^{22}$ N. Park and S. Hong, Phys. Rev. B 72, 045408 (2005).

${ }^{23}$ D. Kang, N. Park, J. Ko, E. Bae, and W. Park, Nanotechnology 16, 1048 (2005) 